\section{Flashing giants forge fluorine}

\section{G. J. Mathews}

FLUIORINF is one of the few chemical elements whose stellar origin is still being debated. There are two reasons for this. For one, the only stable fluorine isotope, ${ }^{19} \mathrm{~F}$, is fragile and easily destroyed by proton capture reactions at stellar temperatures. Most elements of similar mass to fluorine are produced during the late stages of thermonuclear burning in massive stars. However, fluorine is so quickly destroyed that it tends to be bypassed by normal stellar evolution. The second reason is that, although clues to the origin of many elements can be obtained from stellar surface abundances, it has been difficult to find fluorine in stars, owing to a lack of useful atomic fluorine lines in the visible region of the spectrum. This latter difficulty was overcome recently by Jorissen, Smith and Lambert', who obtained fluorine abundances on the surfaces of various red giant stars from the infrared rotation-vibration lines of the $\mathrm{HF}$ molecule. Their measurements con- stitute the only available information on the abundance of fluorine outside the Solar System, and they provide strong evidence that fluorine is produced deep within the interiors of red giant stars during episodic flashes of thermonuclear burning.

Red giant stars are identified according to their spectral class (or redness). In order of increasing redness (or decreasing surface temperature), the types of red giant stars studied in this work were those denoted $\mathrm{K}, \mathrm{Ba}, \mathrm{M}, \mathrm{MS}, \mathrm{S}, \mathrm{Sc}, \mathrm{N}$ and $\mathrm{J}$. These different spectral types can roughly be identified with ages of red giant stars as they evolve along what is called the asymptotic giant branch ${ }^{2}$ (see box). This is a phase in which stars become progressively more luminous and red as thermonuclear burning occurs in shells outside of an inert carbonoxygen core while the outer envelopes become extended. There is a peculiarity in the structure of these $\operatorname{stars}^{2,3}$. Helium burning (by which three helium nuclei

\title{
Asymptotic-giant-branch stars
}

Top, structure of a red giant star as it evolves along the asymptotic giant branch. The outer convective envelope extends to hundreds of times the diameter of the Sun and continuously mixes material from its base to the surface. Below this, a hydrogen-burning shell produces helium (predominantly via the 'CNO' cycle for stars more massive than the Sun). The radius of this shell is actually only a few times the radius of the Earth and would barely be visible if this figure were drawn to scale. This radius, however, encompasses a mass comparable to that of the Sun. Slightly below the hydrogen-burning shell (a fraction of an Earth radius and a thousandth of a solar mass away) is a shell burning helium into carbon. All of this rests on an inert core of mostly carbon plus some oxygen formed during helium burning.

Below, evolution of the helium- and hydrogen-burning shells. Periodically (every 30-100 thousand years, depending upon the mass of the star) the helium shell ignites. Because the star can not expand fast enough to dissipate the heat generated, a thermonuclear runaway ensues. This is arrested by the
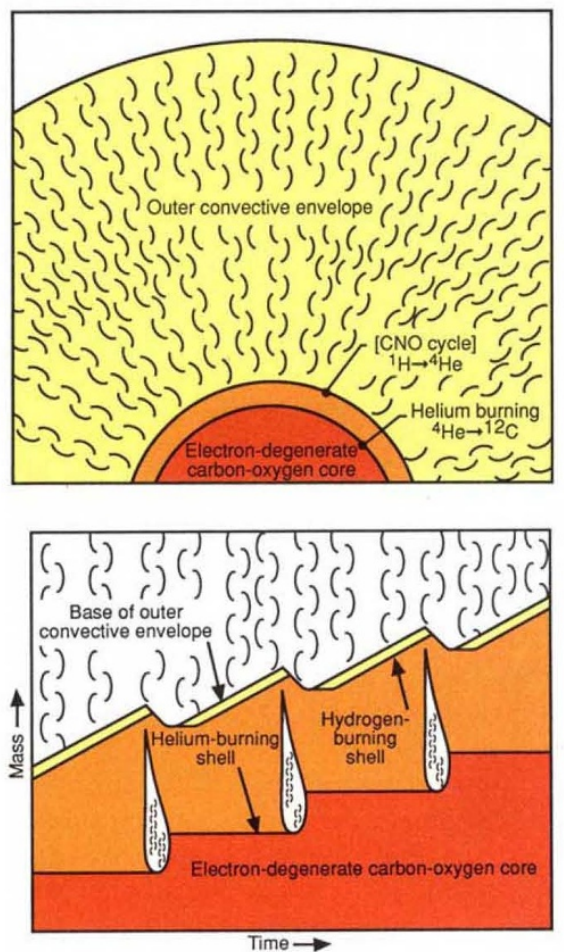
development of a convective shell (teardrop shape in this figure). The hydrogenburning shell then temporarily ceases and the outer convective zone briefly dips into the region below the hydrogen-burning shell. This process dredges up material from the helium-burning regions to the stellar surface. The observations of fluorine enhancement along with carbon on the surface of evolved red giants strongly suggests that fluorine is produced with carbon as a byproduct of helium burning and subsequently dredged to the surface.
G.J.M. are fused into a carbon nucleus) occurs in such a thin shell that as energy is released, the temperature rises much faster than the star can expand and cool. This leads to a thermonuclear runaway which is arrested only by the development of a deep convection zone which dredges material from the thermonuclear burning regions all the way to the surface of the star. This process of episodic helium-burning shell flashes implies that as these stars evolve, their surfaces become enriched in the products of thermonuclear burning.

It is therefore significant that these measurements have revealed fluorine abundances as much as 30 times those in the Sun. This trend can be seen in the graph, taken from Jorissen et al. ${ }^{1}$, which shows the logarithm of the ratio of fluorine to oxygen, $[\mathrm{F} / \mathrm{O}]$, (relative to the mean ratio for $\mathrm{K}$ and $\mathrm{M}$ red giants) as a function of the carbon to oxygen ratio, $\mathrm{C} / \mathrm{O}$. The various symbols represent different spectral types. For example, the open squares show K-stars whose fluorine abundance is not much different from that of the Sun. The more evolved $M$ stars (open circles) show increases in fluorine and carbon abundances relative to oxygen as do $S$ (closed circles) $\mathrm{N}$ (crosses) and $\mathrm{J}$ stars (open triangles). The trend of increasing carbon relative to oxygen with spectral type is an expected outcome of the dredge-up process which brings the carbon ashes of helium burning to the stellar surface. The correlation of increasing fluorine along with increasing carbon strongly suggests that fluorine is also being manufactured by the star.

An important question, however, is how fluorine could be produced and survive at the temperature of the heliumshell flashes. The authors have investigated several possibilities and conclude that the most likely reaction sequence involves the capture of an $\alpha$-particle (helium nucleus) by ${ }^{14} \mathrm{~N}$ to give radioactive ${ }^{18} \mathrm{~F}$, which decays to give ${ }^{18} \mathrm{O}$; proton capture by this then leads indirectly to ${ }^{19} \mathrm{~F}$ - denoted ${ }^{14} \mathrm{~N}(\alpha, \gamma){ }^{18} \mathrm{~F}\left(\beta^{+}\right){ }^{18} \mathrm{O}(\mathrm{p}, \alpha){ }^{15} \mathrm{~N}(\alpha, \gamma){ }^{19} \mathrm{~F}$. The protons are produced by a pair of reactions involving ${ }^{13} \mathrm{C}$ and ${ }^{14} \mathrm{~N}$. The fact that there are only a few protons present and that the circulation removes fluorine from the high-temperature regions saves the fluorine from destruction by proton capture. The fact that fluorine has not been destroyed by the ${ }^{19} \mathrm{~F}(\alpha, \mathrm{p})^{22} \mathrm{Ne}$ reaction also implies a constraint on the temperature and neutron source reaction for the slow neutron-capture (s-process) nucleosynthesis of heavy elements ${ }^{4-6}$. At temperatures high enough to produce neutrons via ${ }^{22} \mathrm{Ne}(\alpha, \mathrm{n})^{25} \mathrm{Mg}$ in heliumshell flashes, fluorine would be destroyed. Thus, ${ }^{22} \mathrm{Ne}$ can not be the neutron source for the s-process. This is NATURE - VOL 359 • 3 SEPTEMBER 1992 